\title{
Diversity Analysis of Rhizosphere Soil Microbial Population Structure and Screening and Identification of Dominant Salt-Tolerant Strains of JunCao"Oasis No. 1"
}

\section{ZhiQi Xiao}

Fujian Agriculture and Forestry University

ZhanXi Lin ( $\square$ 731166321@qq.com)

Fujian Agriculture and Forestry University https://orcid.org/0000-0001-6289-7325

\section{Research Article}

Keywords: Juncao"Oasis No.1", 16SrDNA high-throughput sequencing, Rhizosphere soil microorganism, Pseudomonas fluorescens, Bacillus thuringiensis, Isolation and culture, Colony PCR

Posted Date: February 1st, 2022

DOI: https://doi.org/10.21203/rs.3.rs-1240877/v1

License: (c) (i) This work is licensed under a Creative Commons Attribution 4.0 International License. Read Full License 


\section{Abstract}

Purpose: As a newly cultivated grass species widely used in saline-alkali soil treatment, Juncao"Oasis No.1" can effectively enhance the microbial population abundance in saline-alkali soil. It has a good effect on improving saline-alkali soil.

Methods: In order to further study the microbial community in the rhizosphere soil of Juncao"Oasis No.1" , the dominant strains with important ecological value were screened out. In this paper, soil samples were collected from saline-alkali soil planted with Juncao"Oasis No. 1" and unplanted Juncao"Oasis No. 1", and the microbial populations were sequenced by 16SrDNA high-throughput sequencing, in order to identify the difference of microbial population, the dominant bacteria in rhizosphere soil of Juncao"Oasis No. 1" was selected, and the dominant bacteria with important ecological value were isolated and cultured and identified by Colony PCR.

Results: The results showed that Pseudomonas fluorescens and Bacillus thuringiensis. were the dominant strains with important ecological value in rhizosphere soil of Juncao"Oasis No.1" . After isolation and culture, Colony PCR identification showed that the dominant strain was successfully isolated and cultured.

Conclusions: In this paper, the dominant strains with important ecological value in rhizosphere soil of Juncao" Oasis No. 1" were screened and cultured, which provided theoretical basis for the improvement of saline-alkali soil by using Juncao"Oasis No. 1", it is of great guiding significance.

\section{Introduction}

JunCao refers to a new type of herbaceous plant that can be used for cultivating edible and medicinal fungi after systematic breeding by the threestage systematic screening method of cultivated edible and medicinal fungi(Zhou et al, 2021).JunCao technology was invented in 1987 by Lin Zhanxi, a researcher of mycelia research Institute of Fujian Agriculture and Forestry University. JunCao technology was originally used to replace trees to grow edible and medicinal fungi(Liu et al, 2015). That is, by using fungus instead of trees as the substrate for cultivating edible and medicinal fungi, the prominent "fungus forest contradiction" caused by massive felling of trees for cultivation of edible and medicinal fungi was alleviated.

After the invention of the JunCao technology, it immediately attracted the attention of THE United Nations Development Program and FAO. In 1994, it was listed as a "Priority Cooperation Project between China and Other Developing Countries" by the United Nations Development Program, and in 2017, it was listed as a key project of the United Nations Peace development Fund and promoted to the world. Contribute Chinese wisdom and Chinese plan to the international poverty reduction cause. With the development of The Times, the JunCao technology has been widely used in the field of ecological management, playing an important role in the process of soil salinization control.

JunCao"Oasis No.1", a new grass species selected and cultivated by the fungus technology in recent years, has been widely used in soil salinization control. From 2013 to 2017, Fujian Agriculture and Forestry University National JunCao Engineering Technology Center has carried out experimental studies on planting JunCao"Oasis No. 1" to treat saline-alkali land in several regions of China, and achieved ideal treatment results.

Relevant research data show that the JunCao"Oasis No.1" has developed root system, rapid growth and is rich in various crude proteins, which has a quick and good effect in the treatment of saline-alkali land.Most importantly, planting JunCao"Oasis No. 1" can significantly increase the number of soil microorganisms, thus improving the population structure of soil microorganisms, thus fundamentally improving the soil physical and chemical properties of saline-alkali land, reducing the salinity of saline-alkali soil, and effectively restoring the soil fertility of saline-alkali soil(Zhou et al, 2021).This is also the core mechanism of JunCao "Oasis No.1" to improve saline-alkali soil.

With the development of modern molecular biology technology, high-throughput sequencing technology plays an important role in the detection and identification of microbial populations(Reuter et al, 2015), among which 16SrDNA high-throughput sequencing technology is the most widely used in the identification of microbial populations due to its good specificity. 16SrDNA is located on the small ribosomal subunit of prokaryotic cells(Young et al, 2017), including 10 Conserved Regions and 9 Hypervariable Regions(Goldmann et al, 2021). There is little difference between bacteria in Conserved Regions, and the Hypervariable Regions are genus or species specific(Vezzulli et al, 2018). There are certain differences with the different genetic relationship. Therefore(Luan et al, 2020), 16SrDNA can be used to reveal the characteristic nucleic acid sequence of biological species, and is considered to be the most suitable indicator for bacterial phylogeny and taxonomic identification(Elberri et al, 2020). 16SrDNA Amplicon Sequencing refers to the selection of one or several mutated regions, the design of universal primers for PCR amplification using the conserved regions, and then the Sequencing analysis and species identification of the highly mutated regions(Kosacki et al, 2020). 16SrDNA amplicon sequencing has become an important means to study the composition and structure of microbial communities in environmental samples(Laranjo et al, 2004).

Meanwhile, colony PCR has been widely used in the identification of positive clones(Woodman et al, 2016).Colony PCR directly uses the DNA exposed after thermal hydrolysis of bacterial bodies as a template for PCR amplification, which saves a series of complex processes such as extraction of bacterial body DNA(Asano et al, 2011). Compared with the traditional method of extraction of bacterial body DNA and then PCR amplification, it is more time-saving and labor-saving and suitable for rapid batch identification of bacterial strains(Kong et al, 2005).

Therefore, it is only necessary to design specific amplification primers for dominant strains and detect whether the isolated and cultured strains can generate corresponding specific target gene fragment bands through Colony PCR amplification reaction, so as to determine whether the isolated and

Page 2/18 


\section{Materials And Methods}

\subsection{Soil sample collection}

The Five-point sampling method was used to extract deep soil with a depth of about $20 \mathrm{~cm}$ in the Blank saline-alkali area, where no JunCao"Oasis No. 1 " plants were planted and no plants were growing on the surface, as Blank soil samples. Plant soil was selected in the planting section of JunCao"Oasis No. 1" and the rhizosphere soil of JunCao"Oasis No. 1" with a rhizosphere depth of about $20 \mathrm{~cm}$ was extracted as soil sample of Experimental group (Table 1).

Table 1

Soil sample collection information

\begin{tabular}{|lllll|}
\hline The sample Group & Sampling Method & Sampling Depth & Sample Location & Sample Number \\
\hline Blank soil sample & Five point sampling method & $20 \mathrm{~cm}$ & Blank saline area & K.B.1-K.B.8 \\
\hline Experimental soil sample & Five point sampling method & $20 \mathrm{~cm}$ & Rhizosphere of JunCao & S.Y.1-S.Y.8 \\
\hline
\end{tabular}

\subsection{Medium}

Salt-tolerant Kim B solid medium: Using for isolation culture and purification of dominant Salt tolerance strains in rhizosphere soil of JunCao"Oasis No. 1" plant.

Its formula is: hydrolyzed peptone $20.0 \mathrm{~g}, \mathrm{NaCl} 20.0 \mathrm{~g}, \mathrm{MgSO}_{4} 1.5 \mathrm{~g}, \mathrm{~K}_{2} \mathrm{HPO}_{4} 1.5 \mathrm{~g}$, Agar $15.0 \mathrm{~g}, \mathrm{pH} 7.3 \pm 0.2$.

Solid medium for salt-tolerant Bacillus: Used for isolation culture screening and purification of dominant Salt tolerance strains in rhizosphere soil of JunCao"Oasis No.1" plant.

Its formula is:Glucose $10.0 \mathrm{~g}, \mathrm{NaCl} 20.0 \mathrm{~g}, \mathrm{CaPO}_{4} 5.0 \mathrm{~g},\left(\mathrm{NH}_{4}\right)_{2} \mathrm{SO}_{4} 0.5 \mathrm{~g}, \mathrm{KCl} 0.2 \mathrm{~g},\left(\mathrm{MgSO}_{4}\right) 7 \mathrm{H}_{2} \mathrm{O} \mathrm{0.1 \textrm {g } , \mathrm { MnSO }} 40.0001 \mathrm{~g}, \mathrm{FeSO}{ }_{4} 0.0001 \mathrm{~g}, \mathrm{Yeast}$ extract $0.5 \mathrm{~g}$, Agar $20.0 \mathrm{~g}, \mathrm{pH} 7.0 \pm 0.2$.

\subsection{Main reagent materials}

MP Biomedicals Fast DNA Soil Sample Extraction Kit, 2×Taq Master Mix reagent (containing Taq enzyme, dNTP, Mg ${ }^{2+}$ ), ddH ${ }_{2} \mathrm{O}, 6 \times \mathrm{DNA} \mathrm{Loading}$ Buffer reagent, DL2000 DNA Marker reagent, Glegreen nucleic acid gel dye, 1×TAE gel electrophoresis buffer.

\subsection{Difference analysis of soil microbial population between Blank group and Experimental group}

According to the operating instructions of MP Biomedicals Fast DNA soil sample extraction Kit, total DNA of soil microorganisms in Blank group and Experimental group are extracted, and 8 total DNA samples of soil microorganisms in each group are extracted. The 8 total DNA samples of soil microorganisms in Blank group are numbered as follows: KB1, KB2, KB3, KB4, KB5, KB6, KB7, KB8; The total DNA samples of 8 soil microorganisms in the Experimental group were numbered as SY1, SY2, SY3, SY4, SY5, SY6, SY7 and SY8. After passing the test, the total DNA extracted from soil microorganisms was used as the template to amplify the 16SrDNA fragment of bacteria using PCR technology. After the amplification, the PCR amplification products were recovered and analyzed by computer.

\subsection{Isolation screening and purification of dominant strains from rhizosphere soil of JunCao"Oasis No.1"}

The rhizosphere soil of the JunCao"Oasis No. 1 " plant stored at $-80^{\circ} \mathrm{C}$ of $9.0 \mathrm{~g}$ was weighed and placed in a sterilized $250 \mathrm{ml}$ conical flask. $90 \mathrm{ml}$ physiological saline was added to form a soil suspension with a concentration gradient of $10^{-1}$. After sealing, it was placed in a constant temperature oscillation incubator at $37^{\circ} \mathrm{C}$ and $150 \mathrm{r} / \mathrm{min}$ for $120 \mathrm{~min}$ to make the microorganisms in the soil fully dissolved in physiological saline. And then successively diluted into soil suspensions with concentration gradients of $10^{-2}, 10^{-3}, 10^{-4}, 10^{-5}, 10^{-6}, 10^{-7}, 10^{-8}$ and $10^{-9}(\mathrm{Li}$ et al, 2020).

Soil suspensions with concentration gradients of $10^{-4}, 10^{-5}, 10^{-6}$ and $10^{-7}$ were selected, 100 microliters of soil suspensions were absorbed with a 200 microliter pipette gun and inoculated into bacterial culture dishes containing sterilized TSB medium, which were evenly smeared on the surface of TSB medium with a coating stick. Soil suspensions with each concentration gradient were inoculated into 4 culture dishes. After sealing, pour into a constant temperature incubator at $37^{\circ} \mathrm{C}$ for 3-6 days. 
White and yellow single colonies growing on the surface of TSB medium were respectively picked out after the inoculating ring was burned red and cooled by alcohol lamp. After sterilization for $20 \mathrm{~min}$ at $1 \times 10^{5} \mathrm{~Pa}$, continuous lines were drawn on the TSB medium until all colonies growing on the surface of TSB medium were white and yellow.

\subsection{Colony PCR identification of dominant strains in rhizosphere soil of JunCao"Oasis No. 1"}

The white and yellow single colonies on the surface of TSB medium were picked with the tip of a sterilized pipetting gun. the colonies were placed

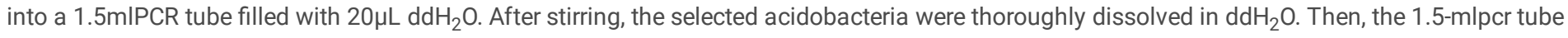
containing the liquid of the dominant strain was covered and placed in an induction cooker with boiling water at $100^{\circ} \mathrm{C}$ for $5 \mathrm{~min}$, so that the dominant strain was fully cracked under heating conditions and DNA was fully exposed(Pesce et al, 2019).

Specific primers for the dominant strain were designed (Table 2) using the DNA of the dominant strain that was fully lysed as the template, corresponding PCR reaction system was configured (Table 3), and appropriate PCR reaction conditions were set (Table 4) for PCR amplification reaction.

Table 2

Design of specific primers for dominant strains

\begin{tabular}{|llll|}
\hline Superiority strains & Upstream primer & Downstream primers & Fragment length \\
\hline Pseudomonas fluorescens & Pse-121L: & Pse-678R: & 557bp \\
& ACGCTAATACCGCATACG & ACTGGTGTTCCTTCCTATATC & \\
Bacillus thuringiensis & Bac-819L: & Bac-1518R: & $699 \mathrm{bp}$ \\
& ACGATGAGTGCTAAGTGT & ATACGGCTACCTTGTTACG & \\
\hline
\end{tabular}

Table 3

Colony PCR reaction system of dominant strains

\begin{tabular}{|ll|}
\hline PCR reaction system & $\mathbf{2 0} \mu \mathrm{L}$ \\
\hline The template & $3 \mu \mathrm{L}$ \\
\hline Upstream primer & $1 \mu \mathrm{L}$ \\
\hline Downstream primers & $1 \mu \mathrm{L}$ \\
$2 \times T a q$ Master Mix & $10 \mu \mathrm{L}$ \\
$\mathrm{ddH}_{2} \mathrm{O}$ & $5 \mu \mathrm{L}$ (Fill the system to $20 \mu \mathrm{L})$ \\
\hline
\end{tabular}

Table 4

Conditions for Colony PCR amplification of dominant strains

\begin{tabular}{|ll|}
\hline Reaction stage & Reaction time \\
\hline $94^{\circ} \mathrm{C}$ Pre degeneration & $1 \mathrm{~min} 30 \mathrm{sec}$ \\
\hline $94^{\circ} \mathrm{C}$ Degeneration & $30 \mathrm{sec}$ \\
\hline $50^{\circ} \mathrm{C}$ Annealing & $30 \mathrm{sec}$ \\
\hline $72^{\circ} \mathrm{C}$ Extension & $1 \mathrm{~min}$ \\
\hline $\mathrm{Cycle}$ Number & 34 \\
\hline $72^{\circ} \mathrm{C}$ Extension & $5 \mathrm{~min}$ \\
\hline $4^{\circ} \mathrm{C}$ Save & Forever \\
\hline
\end{tabular}

\subsection{Gram staining reaction of dominant strains}


The dominant strains were stained with The Gram kit, and a small number of bacteria were selected and evenly smeared on the surface of the slide. After drying under the alcohol lamp, the crystal violet reagent was smeared on the surface of the bacteria for $1 \mathrm{~min}$, and then washed with water. Then apply the iodine solution to the bacteria surface for staining for $1 \mathrm{~min}$, and rinse with water after the staining. Drop appropriate amount of anhydrous ethanol for decolorization for 20 seconds, then rinse with water. Finally, the saffron reagent was applied to the thall surface for dyeing for 1 min, and then washed with water. After drying under an alcohol lamp, the staining results were observed under a microscope(Moyes et al, 2009).

\section{$1.8 \mathrm{Gel}$ electrophoresis reaction of Colony PCR reaction products}

The electrophoresis voltage was $120 \mathrm{~V}$ and the electrophoresis time was 30min. After the electrophoresis, gel electrophoresis imaging system was used to verify the effect of gel electrophoresis

\section{Results And Analysis}

\subsection{Difference analysis of OTUs clustering between Blank group and Experimental group}

The differential distribution of OTUs clustering of soil microbial populations in the Blank group and the Experimental group is shown in Fig. 1. As can be seen from the figure, except for K.B.7 samples in the blank group (which may be caused by the uneven distribution of microbial population in the soil), the OUTs clustering numbers of all samples in the Experimental group were significantly higher than those in the Blank group. This indicated that the abundance of microbial population in the soil samples of the Experimental group was significantly higher than that of the Blank group, and the cultivation of JunCao"Oasis No.1" significantly restored and improved the abundance of microbial population in saline soil.

2.2 Difference analysis of OTUs clustering numbers of microbial populations at different classification levels between blank group and experimental group soil samples

The OTUs clustering numbers of microbial populations at different classification levels of soil samples from the blank group and the experimental group were shown in Fig. 2, Fig. 3 and Table 5. It can be concluded that the OTUs clustering number of microbial population of each soil sample in the experimental group is significantly improved at the level of phylum classification and genus classification compared with that of each soil sample in the blank group. This indicated that the microbial population abundance of the soil samples in the experimental group was significantly improved at the level of Phylum classification, Order classification, Family classification and genus classification, which was also an important reason for the obvious difference in the microbial population structure of the soil samples in the experimental group compared with the soil samples in the blank group. 
Table 5

OTUs clustering numbers of microbial populations at different classification levels of soil samples in Blank group and Experimental group

\begin{tabular}{|llllllll|}
\hline Sample_Name & Kingdom & Phylum & Class & Order & Family & Genus & Species \\
\hline K.B.1 & 28947 & 24740 & 24313 & 17028 & 14105 & 10680 & 1430 \\
\hline K.B.2 & 63888 & 58342 & 57837 & 55278 & 48743 & 46352 & 37711 \\
\hline K.B.3 & 55521 & 46349 & 44564 & 33666 & 23107 & 12643 & 1940 \\
\hline K.B.4 & 55837 & 48571 & 46858 & 33583 & 25159 & 17519 & 1475 \\
\hline K.B.5 & 55480 & 46471 & 44680 & 31394 & 22897 & 13731 & 2847 \\
\hline K.B.6 & 60962 & 51357 & 50217 & 34760 & 26991 & 18492 & 883 \\
\hline K.B.7 & 58570 & 50246 & 48498 & 34045 & 26955 & 16071 & 2791 \\
\hline K.B.8 & 57226 & 47128 & 45398 & 28047 & 19794 & 9443 & 1629 \\
\hline S.Y.1 & 61627 & 53175 & 51108 & 45812 & 37932 & 14202 & 2042 \\
\hline S.Y.2 & 60373 & 49326 & 46868 & 39540 & 30310 & 9482 & 1701 \\
\hline S.Y.3 & 58767 & 50327 & 48938 & 43958 & 38046 & 12535 & 1471 \\
\hline S.Y.4 & 57131 & 49098 & 48290 & 45285 & 41161 & 30101 & 4054 \\
\hline S.Y.5 & 57557 & 49198 & 46649 & 40243 & 32258 & 12862 & 1681 \\
\hline S.Y.6 & 55593 & 48979 & 46952 & 41203 & 34106 & 13295 & 1787 \\
\hline S.Y.7 & 55685 & 47915 & 45874 & 39869 & 32574 & 12025 & 1610 \\
\hline S.Y.8 & 53858 & 46709 & 44925 & 37963 & 30640 & 15860 & 3312 \\
\hline
\end{tabular}

\subsubsection{Analysis of species relative abundance differences at Phylum level}

According to the phylum level relative species abundance histogram (Fig. 4) and Phylum level species clustering heat map (Fig. 5) of soil microbial populations in the Blank group and the Experimental group, the changes in Phylum level relative species abundance between the two groups were analyzed.

It can be seen from Fig. 4 that, at the Phylum level, Proteobacteria and Firmicutes with relatively high population abundance in the Blank group soil samples (K.B.1-K.B.8) decreased significantly in the Experimental group soil samples (R.S.1-R.S.8). While Crenarchaeota, Unidentified Bacteria, Actinobacteriota, Cyanobacteria, Bacteroidota, The relative abundance of phyla such as Chloroflexi did not change much.

The relative species abundance of Acidobacteriota and Desulfobacterota in experimental soil samples (R.S.1-R.S.8) has been effectively increased. The relative abundance of Acidobacteriota in the Blank group (K.B.1-K.B.8) was $1.0 \%, 0.74 \%, 2.7 \%, 2.0 \%, 2.8 \%, 1.6 \%, 2.3 \%$ and $2.2 \%$ respectively. The relative abundance of Acidobacteriota in the Experimental group (S.Y.1-S.Y.8) was 5.5\%, 8.4\%, 5.0\%, 6.4\%, 5.1\%,6.1\%, 6.1\% and 6.5\% respectively. The relative species abundance of Desulfobacterota in the Blank group (K.B.1-K.B.8) is $0.1 \%, 0.1 \%, 0.4 \%, 0.3 \%, 0.7 \%, 0.6 \%, 0.8 \%$ and $0.7 \%$ respectively. The relative abundance of Desulfobacterota in the Experimental group soil samples (S.Y.1-S.Y.8) is 3.9\%, 5.1\%, 2.2\%, 1.2\%, 5.0\%, 9.5\%, 3.7\% and 2.6\% respectively. The relative abundance of both was significantly increased (Table 6-1, Table 6-2). 
Table 6.1

Relative abundance values of species at the taxonomic level of each sample Phylum

\begin{tabular}{|llllll|}
\hline Taxonomy & Proteobacteria & Crenarchaeota & unidentified_Bacteria & Firmicutes & Desulfobacterota \\
\hline K.B.1 & 0.315268 & 0.14791 & 0.128256 & 0.073955 & 0.001105 \\
\hline K.B.2 & 0.480587 & 0.080311 & 0.050915 & 0.170535 & 0.001554 \\
\hline K.B.3 & 0.184594 & 0.078135 & 0.234162 & 0.056615 & 0.004905 \\
\hline K.B.4 & 0.244525 & 0.139585 & 0.16228 & 0.043558 & 0.003454 \\
\hline K.B.5 & 0.26487 & 0.074162 & 0.196408 & 0.043834 & 0.017893 \\
\hline K.B.6 & 0.258515 & 0.222556 & 0.151572 & 0.033022 & 0.006321 \\
\hline K.B.7 & 0.291434 & 0.054439 & 0.188636 & 0.048566 & 0.032884 \\
\hline K.B.8 & 0.30266 & 0.031054 & 0.217755 & 0.020587 & 0.023731 \\
\hline S.Y.1 & 0.219965 & 0.206321 & 0.159827 & 0.015509 & 0.039206 \\
\hline S.Y.2 & 0.155889 & 0.142453 & 0.155302 & 0.010881 & 0.051364 \\
\hline S.Y.3 & 0.212332 & 0.275509 & 0.13513 & 0.008325 & 0.022211 \\
\hline S.Y.4 & 0.332055 & 0.033679 & 0.093679 & 0.023903 & 0.012124 \\
\hline S.Y.5 & 0.235406 & 0.120587 & 0.193817 & 0.003454 & 0.050363 \\
\hline S.Y.6 & 0.206183 & 0.129706 & 0.171192 & 0.017651 & 0.095509 \\
\hline S.Y.7 & 0.217478 & 0.16038 & 0.182245 & 0.014197 & 0.037617 \\
\hline S.Y.8 & 0.253782 & 0.100104 & 0.161071 & 0.046908 & 0.026321 \\
\hline
\end{tabular}

Table 6.2

Relative abundance values of species at the taxonomic level of each sample Phylum

\begin{tabular}{|lllllll|}
\hline Taxonomy & Acidobacteriota & Actinobacteriota & Cyanobacteria & Bacteroidota & Chloroflexi & Others \\
\hline K.B.1 & 0.010535 & 0.052919 & 0.038204 & 0.016718 & 0.015648 & 0.199482 \\
\hline K.B.2 & 0.007427 & 0.044491 & 0 & 0.002556 & 0.029361 & 0.132263 \\
\hline K.B.3 & 0.027496 & 0.042211 & 0.001382 & 0.015682 & 0.047358 & 0.307461 \\
\hline K.B.4 & 0.020138 & 0.034059 & 0.038722 & 0.01399 & 0.048256 & 0.251434 \\
\hline K.B.5 & 0.028428 & 0.045492 & 0.00038 & 0.009326 & 0.04038 & 0.278826 \\
\hline K.B.6 & 0.016615 & 0.030984 & 0.000622 & 0.008946 & 0.031883 & 0.238964 \\
\hline K.B.7 & 0.043903 & 0.045216 & 0.000898 & 0.007599 & 0.030294 & 0.256131 \\
\hline K.B.8 & 0.033299 & 0.034922 & 0.000622 & 0.007945 & 0.04 & 0.287427 \\
\hline S.Y.1 & 0.055959 & 0.016511 & 0.000864 & 0.00715 & 0.033955 & 0.244732 \\
\hline S.Y.2 & 0.084111 & 0.020449 & 0.000553 & 0.005216 & 0.044491 & 0.329292 \\
\hline S.Y.3 & 0.049085 & 0.013022 & 0.003212 & 0.012746 & 0.020725 & 0.247703 \\
\hline S.Y.4 & 0.064525 & 0.07361 & 0.058826 & 0.051364 & 0.033437 & 0.222798 \\
\hline S.Y.5 & 0.05133 & 0.022003 & 0.00494 & 0.007599 & 0.044525 & 0.265976 \\
\hline S.Y.6 & 0.061278 & 0.031088 & 0.000898 & 0.010501 & 0.031952 & 0.244041 \\
\hline S.Y.7 & 0.061727 & 0.019447 & 0.001934 & 0.009983 & 0.031917 & 0.263074 \\
\hline S.Y.8 & 0.065112 & 0.037858 & 0.006218 & 0.018895 & 0.04 & 0.243731 \\
\hline
\end{tabular}

As can be seen from Fig. 5, at the level of Phylum classification, Verrucomicrobiota, Entotheonellaeota, Thermoplasmata Myxococcota, Desulfuromondia (desulfurization bacterium door), Myxococcota, Acidobacteriota (acid bacillus door), The species clustering of Latescibacterota was significantly higher than that of blank soil samples (K.B.1-K.B.8). 
Desulfobracterota and Acidobacteriae are the dominant Phylum in the Experimental group soil samples (S.Y. 1-S.Y. 8) combined with the column chart of relative species abundance at Phylum level and the heat map of Phylum level clustering.

\subsubsection{Analysis of species relative abundance differences at Order level}

According to the Order level relative species abundance histogram (Fig. 6) and Order level species clustering heat map (Fig. 7) of soil microbial populations in the Blank group and the Experimental group, the changes in Order level relative species abundance between the two groups were analyzed.

It can be seen from Fig. 6 that the relative abundance of species at Order level showed obvious differentiation between samples from Blank group (K.B.1-K.B.8) and Experimental group (S.Y. 1-S.Y. 8). The relative species abundance of experimental soil samples (S.Y. 1-S.Y. 8) at Order level was significantly higher than that of blank soil samples (K.B.1-K.B.8).

The relative species abundance of Rhodospirillales, Sphingomonadales and Burkholderiales in the Experimental group (S.Y. 1-S.Y. 8) was significantly higher than that in the Blank group (K.B.1-K.B.8). The relative abundance of Rhodospirillales in experimental soil samples (S.Y. 1-S.Y. 8) was 4.2\%, $6.1 \%, 4.4 \%, 3.3 \%, 5.1 \%, 4.4 \%, 5.9 \%, 3.6 \%$ respectively. The relative species abundance of Sphingomonadales in Experimental group soil samples (R.S.1-R.S.8) was $0.6 \%, 0.6 \%, 0.7 \%, 6.6 \%, 1.4 \%, 0.7 \%, 0.6 \%, 0.9 \%$ respectively, and in Blank group soil samples K.B.3, K.B.4, K.B.5. The relative abundance of Sphingomonadales was 0 , and the relative abundance of Sphingomonadales in the other Blank groups was extremely low. The relative species abundance of Burkholderiales in experimental soil samples (R.S.1-R.S.8) was $6.2 \%, 3.8 \%, 6.3 \%, 10.9 \%, 4.9 \%, 4.8 \%, 5.2 \%, 4.9 \%$ respectively. Compared with the Blank group (K.B.1-K.B.8), the relative abundance of Rhodospirillales, Sphingomonadales and Burkholderiales in the Experimental group (S.Y. 1-S.Y. 8) was increased (Table 7).

Table 7

Relative abundance of dominant flora species at soil sample Order level in Experimental group

\begin{tabular}{|c|c|c|c|c|c|c|}
\hline Taxonomy & Burkholderiales & Nitrosopumilales & Paenibacillales & Rhodospirillales & unidentified_Desulfuromonadia & Sphingomonadales \\
\hline K.B.1 & 0.001796 & 0.144525 & 0.000587 & 0.002453 & 0.000622 & 0.001209 \\
\hline K.B.2 & 0.411952 & 0.080138 & 0.130432 & 0 & 0.000829 & 0.033472 \\
\hline K.B.3 & 0.002349 & 0.072021 & 0 & 0.004111 & 0.001934 & 0.000794 \\
\hline K.B.4 & 0.002487 & 0.134853 & 0.001105 & 0.002383 & 0.001762 & 0.000484 \\
\hline K.B.5 & 0.004387 & 0.071641 & 0.000104 & 0.005838 & 0.016442 & 0.000415 \\
\hline K.B.6 & 0.002038 & 0.215648 & 0.000104 & 0.002591 & 0.004767 & 0.000311 \\
\hline K.B.7 & 0.018549 & 0.050708 & 0.000207 & 0.009223 & 0.024905 & 0.005389 \\
\hline K.B.8 & 0.006287 & 0.028566 & 0.000138 & 0.006183 & 0.018618 & 0.001174 \\
\hline S.Y.1 & 0.062142 & 0.205803 & 0.000104 & 0.042798 & 0.025941 & 0.005838 \\
\hline S.Y. 2 & 0.037686 & 0.141623 & 0.000138 & 0.061036 & 0.042832 & 0.002453 \\
\hline S.Y.3 & 0.063005 & 0.274577 & 0.000207 & 0.044283 & 0.0162 & 0.005043 \\
\hline S.Y. 4 & 0.108601 & 0.03323 & 0.003351 & 0.021209 & 0.007634 & 0.065596 \\
\hline S.Y.5 & 0.04943 & 0.119931 & 0.000242 & 0.051054 & 0.035959 & 0.013748 \\
\hline S.Y.6 & 0.048394 & 0.127288 & 0.00076 & 0.044352 & 0.084421 & 0.006701 \\
\hline S.Y.7 & 0.052435 & 0.157686 & 0.000173 & 0.059413 & 0.026736 & 0.005561 \\
\hline S.Y.8 & 0.049257 & 0.09658 & 0.000415 & 0.036408 & 0.017375 & 0.009119 \\
\hline
\end{tabular}

It can be clearly seen from Fig. 7 that Rhodospirillales, Rhizobiales, Sphingomonadales, Pseudomondales in soil samples of Experimental group (S.Y. 1-S.Y. 8), The species clustering of Micrococcales was significantly higher than that of blank soil samples (K.B.1-K.B.8). Pseudomondales is one of the most important growth-promoting bacteria in plant rhizosphere.

Combined with the histogram of relative abundance of species at Order level and the heat map of cluster of species at Order level, Pseudomondales and Sphingomonadales were dominant in the soil samples of Experimental group (S.Y. 1-S.Y. 8).

\subsubsection{Analysis of species relative abundance differences at Family level}

According to the Family level relative species abundance histogram (Fig. 8) and Family level species clustering heat map (Fig. 9) of soil microbial populations in the Blank group and the Experimental group, the changes in Family level relative species abundance between the two groups were

Page $8 / 18$ 
analyzed.

As can be seen from Fig. 8, the differences in relative species abundance at Family level between samples from Blank group (K.B.1-K.B.8) and Experimental group (S.Y. 1-S.Y. 8) were more obvious. In experimental soil samples (S.Y.1-S.Y.8), Magnetospiraceae, Sphingomonadaceae, Oxalobacteraceae, The relative species abundance of Desulfuromonadaceae is significantly higher than that of blank soil samples (K.B.1-K.B.8). The relative species abundance of Magnetospiraceae in experimental soil samples (S.Y. 1-S.Y. 8) was 4.0\%, 5.9\%, 4.2\%, 2.0\%, 4.8\%, 4.2\%, 5.7\%, 3.4\% respectively. The relative species abundance of Sphingomonadaceae in experimental soil samples (S.Y. $1-\mathrm{S} . \mathrm{Y} .8$ ) was $0.6 \%, 0.5 \%, 0.5 \%, 6.5 \%, 1.4 \%$, $0.7 \%, 0.6 \%, 0.9 \%$ respectively. The relative abundance of Sphingomonadaceae was 0 in K.B.3,K.B.4,K.B.5 and K.B.6 samples of Blank group, while the relative abundance of Sphingomonadaceae was extremely low in other samples. The relative species abundance of Oxalobacteraceae in Experimental group soil sample (S.Y. 1-S.Y. 8) was $0.1 \%, 0.1 \%, 0.3 \%, 7.8 \%, 0.1 \%, 0.3 \%, 0.3 \%, 0.4 \%$ respectively, and in Blank group soil sample (K.B.1K.B.8), The relative abundance of Oxalobacteraceae was 0 . The relative species abundance of Desulfuromonadaceae in Experimental group soil samples (S.Y. 1-S.Y. 8) was $2.0 \%, 4.1 \%, 1.2 \%, 0.6 \%, 3.2 \%, 8.1 \%, 2.3 \%$ and $1.4 \%$ respectively. (Table 8 ).

Table 8

Relative abundance of dominant flora species at soil sample Family level in Experimental group

\begin{tabular}{|c|c|c|c|c|c|}
\hline Taxonomy & Magnetospiraceae & Paenibacillaceae & Sphingomonadaceae & Desulfuromonadaceae & Oxalobacteraceae \\
\hline K.B.1 & 0.002383 & 0.000587 & 0.001209 & 0 & 0.000415 \\
\hline K.B.2 & 0 & 0.130432 & 0.033472 & 0 & 0 \\
\hline K.B.3 & 0.003282 & 0 & 0 & 0 & 0 \\
\hline K.B.4 & 0.002038 & 0.001105 & 0 & 0 & 0.000484 \\
\hline K.B.5 & 0.005285 & 0.000104 & 0 & 0.001002 & 0 \\
\hline K.B.6 & 0.002314 & 0.000104 & 0 & 0 & 0.000449 \\
\hline K.B.7 & 0.00867 & 0.000207 & 0.005389 & 0.004525 & 0.002867 \\
\hline K.B.8 & 0.005596 & 0.000138 & 0.001174 & 0.001727 & 0.000794 \\
\hline S.Y.1 & 0.040207 & 0.000104 & 0.005838 & 0.019206 & 0.001105 \\
\hline S.Y.2 & 0.058756 & 0.000138 & 0.002453 & 0.040794 & 0.001451 \\
\hline S.Y.3 & 0.042073 & 0.000207 & 0.005043 & 0.012055 & 0.002556 \\
\hline S.Y.4 & 0.019793 & 0.003351 & 0.065596 & 0.005907 & 0.078342 \\
\hline S.Y.5 & 0.048221 & 0.000242 & 0.013748 & 0.032332 & 0.000967 \\
\hline S.Y.6 & 0.042383 & 0.00076 & 0.006701 & 0.080587 & 0.002694 \\
\hline S.Y.7 & 0.057582 & 0.000173 & 0.005561 & 0.023247 & 0.002832 \\
\hline S.Y.8 & 0.034059 & 0.000415 & 0.009119 & 0.014128 & 0.003972 \\
\hline
\end{tabular}

Desulfuromonadaceae,Pseudomonadaceae, Sphingomonadaceae in the Experimental group soil sample (S.Y.1-S.Y.8) can be clearly seen from Fig. 9. The species clustering of Acidithiobacillaceae was significantly higher than that of the Blank group (K.B.1-K.B.8). Pseudomonadaceae is one of the most important growth-promoting bacteria in plant rhizosphere.

Combined with the histogram of relative abundance of species at Family level and the cluster heat map of species at Family level,

Sphingomonadaceae, Pseudomonadaceae and Desulfuromonadaceae are the dominant Family in the experimental group soil samples (R.S.1-R.S.8).

\subsubsection{Analysis of species relative abundance differences at Genus level}

According to the Genus level relative species abundance histogram (Fig. 10) and Genus level species clustering heat map (Fig. 11) of soil microbial populations in the Blank group and the Experimental group, the changes in Genus level relative species abundance between the two groups were analyzed.

The differences in species relative abundance between samples from Blank group (K.B.1-K.B.8) and Experimental group (S.Y. 1-S.Y. 8) were most obvious at Genus level. Fig. 10 shows that species relative abundance of Sphingomonas, Paenarthrobacter, Paenibacillus and Pseudomonas in Experimental soil sample (S.Y. 1-S.Y.8) is higher than that in blank soil sample (K.B.1-K.B.8). The relative abundance of Sphingomonas in experimental soil samples (S.Y. 1-S.Y. 8) was $0.3 \%, 0.3 \%, 0.3 \%, 5.7 \%, 0.5 \%, 0.5 \%, 0.3 \%, 0.6 \%$ respectively. While that in Blank sample K.B.3,K.B.4, K.B.5,K.B.6, K.B.8 was 0 . The relative abundance of species in the other Blank groups was extremely low. 
The relative abundance of Pseudomonas in experimental soil samples (S.Y. 1-S.Y. 8) was 1.7\% in S.Y.1, followed by 1.0\% in S.Y.5. The relative abundance of species in S.Y.4, S.Y.6, S.Y.7, and S.Y.8 were about $0.7 \%, 0.5 \%, 0.8 \%$, and $0.8 \%$ respectively. The relative abundance of species in K.B.1 and K.B.2 samples of Blank group was 0 , and the relative abundance of species in other Blank group samples was extremely low.

The relative species abundance of Paenibacillus in Experimental sample (S.Y. 1-S.Y. 8) was 0.3\% in S.Y.4, while that in Blank sample K.B.1,K.B.3, K.B.5,K.B.6, K.B.7,K.B.8 was 0. The relative abundance of Paenibacillus species in K.B.2 was due to the heterogeneity of soil microbial population distribution (Table 9).

Table 9

Relative abundance of dominant flora species at soil sample Genus level in Experimental group

\begin{tabular}{|lllll|}
\hline Taxonomy & Paenibacillus & Sphingomonas & Paenarthrobacter & Pseudomonas \\
\hline K.B.1 & 0 & 0.001105 & 0 & 0 \\
\hline K.B.2 & 0.130432 & 0.033472 & 0 & 0 \\
\hline K.B.3 & 0 & 0.000484 & 0 & 0.001865 \\
\hline K.B.4 & 0.001105 & 0.000415 & 0 & 0.001762 \\
\hline K.B.5 & 0 & 0.000104 & 0.000173 & 0.000345 \\
\hline K.B.6 & 0 & 0.000242 & 0 & 0.00152 \\
\hline K.B.7 & 0 & 0.00487 & 0.001934 & 0.001796 \\
\hline K.B.8 & 0 & 0.00076 & 0.000484 & 0.000898 \\
\hline S.Y.1 & 0.000104 & 0.002902 & 0.00076 & 0.017168 \\
\hline S.Y.2 & 0.000138 & 0.000829 & 0.000518 & 0.00494 \\
\hline S.Y.3 & 0.000173 & 0.00228 & 0.001071 & 0.00677 \\
\hline S.Y.4 & 0.00304 & 0.056718 & 0.02829 & 0.006874 \\
\hline S.Y.5 & 0.000242 & 0.00456 & 0.00114 & 0.010501 \\
\hline S.Y.6 & 0.000725 & 0.004836 & 0.002487 & 0.005492 \\
\hline S.Y.7 & 0.000173 & 0.003212 & 0.002453 & 0.007634 \\
\hline S.Y.8 & 0.000276 & 0.00601 & 0.004076 & 0.007703 \\
\hline
\end{tabular}

As can be seen from Fig. 11, the species clustering of Sphingomonas and Pseudomonas in the Experimental group (S.Y.1-S.Y.8) was significantly higher than that in the Blank group (K.B.1-K.B.8). Pseudomonas is an important growth-promoting bacterium in plant rhizosphere. At the same time, Staphylococcus was distributed in soil samples of both the Blank group and the Experimental group. Although Staphylococcus was a common pathogenic bacterium in soil, it was distributed in soil samples of both the Blank group and the Experimental group due to its good salt tolerance. Therefore, Staphylococcus is a common strain of soil samples of Blank group and Experimental group.

Combined with the histogram of relative abundance of species at the Genus level and the cluster heat map of species at the Genus level, Sphingomonas, Paenibacillus and Pseudomonas were the dominant species at the Genus level of soil samples from the Gxperimental group (S.Y. 1S.Y.8). Staphylococcus was a common strain of soil samples from Blank group and Experimental group.

\subsection{Ternaryplot analysis}

In Order to find the differences of dominant Species among the three groups of samples at each classification level (Phylum, Class, Order, Family, Genus), the top 10 Species with average abundance at each classification level were selected to generate a Ternaryplot. In order to intuitively check the differences of dominant species among the three groups of samples at different classification levels(Hong et al, 2021). Ternaryplot analysis using the R software VCD Ternaryplot command(Brzosko et al, 2021). The three vertices in the figure represent three sample groups, and the circle represents species. The size of the circle is proportional to the relative abundance. The closer the circle is to the vertex, the higher the content of this species in this group(Marć et al, 2020). Here, the three classification levels with the largest difference in microbial population structure between the Experimental group and the Blank group were selected for comparative analysis: Phylum classification level, Order classification level and Genus classification level.

\subsubsection{Ternaryplot analysis at the classification level of each sample Phylum}

Page $10 / 18$ 
Ternaryplot analysis of classification level of each sample Phylum is shown in Fig. 12. Content of Desulfobacterota and Acidobacteriota in the samples of the Experimental group is significantly higher than that in the samples of the Blank group. Desulfobacterota and Acidobacteriota are the dominant genuses in each sample in the Experimental group.

\subsubsection{Ternaryplot analysis at the classification level of each sample Order}

Ternaryplot analysis of classification level of each sample Order is shown in Fig. 13. Desulfuromonadia and Rhodospirillales content in the samples of the Experimental group is significantly higher than that in the samples of the Blank group.

In addition, the content of Sphingomonadales in S.Y.4 was significantly higher than that in other Experimental groups, indicating that the content of Sphingomonadales reached the highest level in S.Y.4.

Desulfuromonadia, Rhodospirillales and Sphingomonadales were the dominant species in the Experimental group.

\subsubsection{Ternaryplot analysis at the classification level of each sample Genus}

Ternaryplot analysis of classification level of each sample Genus is shown in Fig. 14. The content of Sphingomonas in all samples in the Experimental group is significantly higher than that in the Blank group. The results showed that the dominant bacteria in the Experimental group was Sphingomonas.

\subsection{Determine the target strain for isolation and culture}

Desulfuromondia and Acidobacteriae are the dominant genuses at the Phylum classification level in the experimental group soil samples (R.S.1R.S.8). The dominant bacteria at the Order classification level were Rhodospirillales, Pseudomondales and Sphingomonadales. The dominant genuses at the Family classification level are Sphingomonadaceae, Pseudomonadaceae and Desulfuromonadaceae. The dominant bacteria at the Genus classification level were Sphingomonas, Paenibacillus and Pseudomonas.

Pseudomonas and Bacillus are important growth-promoting bacteria in plant rhizosphere and have good characteristics of salt and alkali tolerance and pest elimination. Therefore, Pseudomonas and Bacillus were selected as the target strains for isolation and culture based on the ecological value of soil salinization control and the ecological function of the strains themselves.

\subsection{Gram staining reaction analysis of target strains}

Gram staining results of Pseudomonas strain are shown in Fig. 15. It can be seen from the figure that the bacteria are elongated rods and stained red, indicating that the strain is Gram-negative. At the same time, the strain gave out visible fluorescence under uv light irradiation of $365 \mathrm{~nm}$ (Fig. 16), which proved that the Pseudomonas strain was Pseudomonas fluorescens.

Gram staining reaction results of bacillus strain are shown in Fig. 17. It can be seen from the figure that the bacterium is elongated rod shaped and stained purple, indicating that the strain is Gram-positive bacterium. Under the microscope, it was found that the strain could produce a large number of rhomboid spore crystal (Fig. 17), which proved that the bacillus strain was Bacillus thuringiensis.

\subsection{Colony PCR gel electrophoresis analysis}

The gel electrophoresis images of PCR reaction products of Pseudomonas fluorescens and Bacillus thuringiensis colonies are shown in Fig. 18. It could be seen that the PCR target bands of Pseudomonas fluorescens colony (557bp) and Bacillus thingiensis colony (699bp) were clear and bright, neatly arranged, and the length and distribution location of the target bands were accurate. Therefore, it can be determined that the target strains isolated from the rhizosphere soil of "Oasis 1" are pseudomonas fluorescens and Bacillus thuringiensis strains.

\section{Results}

In this paper, 16SrDNA high-throughput sequencing technology was used to sequence the microbial population structure of blank soil samples (K.B.1K.B.8) and experimental soil samples (R.S.1-R.S.8). The relative abundance columns and species clustering of Phylum,Order,Family and Genus were determined. Desulfuromondia and Acidobacteriae are the dominant genuses at the Phylum classification level in the Experimental group soil samples (R.S.1-R.S.8). The dominant bacteria at the Order classification level were Rhodospirillales, Pseudomondales and Sphingomonadales. The dominant genuses at the Family classification level are Sphingomonadaceae, Pseudomonadaceae and Desulfuromonadaceae. The dominant bacteria at the Genus classification level were Sphingomonas, Paenibacillus and Pseudomonas. Finally, Pseudomonas fluorescens and Bacillus thuringiensis were isolated and cultured from the rhizosphere soil of JunCao"Oasis No. 1".

\section{Discuss}

As a new grass species, JunCao"Oasis No. 1" can effectively control soil salinization. Pseudomonas fluorescens and Bacillus thuringiensis have a high abundance in rhizosphere soil. Pseudomonas fluorescens and Bacillus thuringiensis are important growth-promoting bacteria in plant

Page $11 / 18$ 
rhizosphere(Rojas-Ruiz et al, 2015). Both of which can synthesize and secrete a large number of insecticidal toxins(Durán et al., 2021).It plays an important role in plant resistance to pests(Shahid et al., 2021).Therefore, current studies on Pseudomonas fluorescens and Bacillus thuringiensis are focused on the field of resistance to disease and insect pests, and there are very few studies on their salt and alkali resistance(Huang et al, 2007).Studies on the salt and alkali resistance of Pseudomonas fluorescens and Bacillus thuringiensis are conducive to fully exploring the great application potential of Pseudomonas fluorescens and Bacillus thuringiensis in environmental protection and industrial production(HernándezPacheco et al, 2021).

In this paper, purified Pseudomonas fluorescens and Bacillus thuringiensis strains were successfully isolated from the rhizosphere soil of JunCao"Oasis No.1" plant with abundant pseudomonas fluorescens and Bacillus thuringiensis species by using Colony PCR identification method. It laid a solid theoretical foundation for the subsequent saline-alkali resistance of Pseudomonas fluorescens and Bacillus thuringiensis.

At the same time, it is also confirmed from the side that the mycelium JunCao"Oasis No. 1" plant has a good saline-alkali soil control function, which provides a new method and idea for the treatment of saline-alkali soil by biological methods, and has a good practical guiding significance and application value.

\section{Declarations}

\section{Statement of funding and informed consent}

The fund of this paper is provided by the National Engineering Technology Research Center of JunCao, Fujian Agriculture and Forestry University. The project is supported by the Interdisciplinary integration to promote the high-quality development of JunCao science and industry (XKJC712021030) and the Key technology Research and application of Mycelia Germplasm Innovation and Industrial Utilization of Fujian Province (2021NZ0101).

As the corresponding author of this article, I hereby declare:

XiaoZhiqi, the first author of this paper, is my student, who is responsible for the data analysis and article writing of this paper. I have planned and communicated with the first author for many times about the fund operation and various writing work of this paper, and I am fully aware of it.

This paper was written and submitted with my full knowledge and communication with the first author. Accurate data analysis, capital operation in place. Meet the journal submission standards.

\section{Ethical Declarations}

I declare that this paper is the research result of data analysis and corresponding writing completed by my student XiaoZhiqi under my full knowledge and guidance.

The data in this paper are all from experiments, and all experimental data are clear and accurate. It does not include any research results published or written by others, and there is no plagiarism. If found to have infringed upon the intellectual property rights of others, he/she shall bear due responsibilities.

\section{The author contributions}

I declare that the first author of this thesis is XiaoZhiqi, who as my student is mainly responsible for the development of the thesis experiment, the analysis of the experimental data of the thesis and the compilation of the thesis.

As the corresponding author, I am mainly responsible for the final revision and submission guidance of this thesis.

\section{Data availability description}

I declare that all the experimental data in this paper are obtained from the experiment of XiaoZhiqi, the first author of this paper, the data is true and accurate, all available.

The data related to this paper has not been uploaded to a fixed data repository and will be submitted as a separate file. All data in this paper are true and reliable, and do not include any research results published or written by others. There is no plagiarism, and I am fully aware of it. If found to have infringed upon the intellectual property rights of others, he/she shall bear due responsibilities.

\section{Consent to publication}

I declare that I am the corresponding author of this paper. Xiao Zhiqi, the first author of this paper and I agree that this paper will be published and published in the Annual Journal of Microbiology if it meets the publication requirements.

\section{Statement of Competitive interest}


As the corresponding author of this paper, I hereby declare that I have not had any interest dispute or interest competition with anyone or any institution in the process of experiment and compilation of this paper. The funds for the experiment are from the special research funds of the university, and no individual donation is required.

\section{Acknowledgements}

As the corresponding author of this paper, I would like to thank the first author of this paper, my student Xiao Zhiqi. He is the data analyst and author of this paper. For this paper's overall conception and saved to write made a great contribution!

At the same time, I also want to thank my colleagues, who have provided many valuable references for the analysis of the data and the compilation of the paper. Finally, I would like to thank the editors of Annals of Microbiology for reviewing my paper in their busy schedule. I hope the paper can be published smoothly!

\section{Conflicts of interest and acknowledgements}

I hereby declare that there is no conflict of interest with any individual or institution in the experiment and writing process of this paper, and there is no risk of any conflict of interest. In the process of experiment, my student Xiao Zhiqi paid hard work, he is the actual author and the first author of this paper, and his colleague is also the direct analysis of experimental data. For this, I would like to extend my solemn thanks to my student Xiao Zhiqi!

\section{References}

1. Asano, R., Kubori, K., Ozutsumi, Y., Yamamoto, N., Otrawa, K., Nakai, Y., 2011. Detection of Escherichia coli in a cattle manure composting process by selective cultivation and colony polymerase chain reaction. J. of Env. Sc. \& Hlth., Part B 46, 122-127.

https://doi.org/10.1080/03601234.2011.534972

2. Brzosko, E., Bajguz, A., Chmur, M., Burzyńska, J., Jermakowicz, E., Mirski, P., Zieliński, P., 2021. How Are the Flower Structure and Nectar Composition of the Generalistic Orchid Neottia ovata Adapted to a Wide Range of Pollinators? IJMS 22, 2214. https://doi.org/10.3390/ijms22042214

3. Durán, D., Bernal, P., Vazquez-Arias, D., Blanco-Romero, E., Garrido-Sanz, D., Redondo-Nieto, M., Rivilla, R., Martín, M., 2021. Pseudomonas fluorescens F113 type VI secretion systems mediate bacterial killing and adaption to the rhizosphere microbiome. Sci Rep 11, 5772. https://doi.org/10.1038/s41598-021-85218-1

4. Elberri, A.I., Galal-Khallaf, A., Gibreel, S.E., El-Sakhawy, S.F., El-Garawani, I., El-Sayed Hassab ElNabi, S., Mohammed-Geba, K., 2020. DNA and eDNA-based tracking of the North African sharptooth catfish Clarias gariepinus. Molecular and Cellular Probes 51, 101535. https://doi.org/10.1016/j.mcp.2020.101535

5. Goldmann, T., Hillemann, D., Maurer, F., Kalsdorf, B., Krupar, R., Stellmacher, F., Perner, S., 2021. Mycobacterium szulgai als Posititivkontrolle zur Detektion von Kontaminationen beim Nachweis des Mycobacterium tuberculosis-Komplexes durch eine spezifische 16S-rDNA-PCR an FFPEMaterial. Pathologe 42, 83-85. https://doi.org/10.1007/s00292-021-00912-1

6. Hernández-Pacheco, C.E., Orozco-Mosqueda, M. del C., Flores, A., Valencia-Cantero, E., Santoyo, G., 2021. Tissue-specific diversity of bacterial endophytes in Mexican husk tomato plants (Physalis ixocarpa Brot. ex Horm.), and screening for their multiple plant growth-promoting activities. Current Research in Microbial Sciences 2, 100028. https://doi.org/10.1016/j.crmicr.2021.100028

7. Hong, J., Moon, H., Kim, J., Lee, B., Kim, G.-B., Lee, H., Kim, Y., 2021. Differentiation of carbon black from black carbon using a ternary plot based on elemental analysis. Chemosphere 264, 128511. https://doi.org/10.1016/j.chemosphere.2020.128511

8. Huang, K., Badger, M., Haney, K., Evans, S.L., 2007. Large scale production of Bacillus thuringiensis PS149B1 insecticidal proteins Cry34Ab1 and Cry35Ab1 from Pseudomonas fluorescens. Protein Expression and Purification 53, 325-330. https://doi.org/10.1016/j.pep.2007.01.010

9. Kong, P., Richardson, P.A., Hong, C., 2005. Direct colony PCR-SSCP for detection of multiple pythiaceous oomycetes in environmental samples. Journal of Microbiological Methods 61, 25-32. https://doi.org/10.1016/j.mimet.2004.10.019

10. Kosacki, J., Boisset, S., Maurin, M., Cornut, P.-L., Thuret, G., Hubanova, R., Vandenesch, F., Carricajo, A., Aptel, F., Chiquet, C., 2020. Specific PCR and Quantitative Real-Time PCR in Ocular Samples from Acute and Delayed-Onset Postoperative Endophthalmitis. American Journal of Ophthalmology 212, 34-42. https://doi.org/10.1016/j.ajo.2019.11.026

11. Laranjo, M., Machado, J., Young, J.P.W., Oliveira, S., 2004. High diversity of chickpea Mesorhizobium species isolated in a Portuguese agricultural region. FEMS Microbiology Ecology 48, 101-107. https://doi.org/10.1016/j.femsec.2003.12.015

12. Li, Y.-J., Li, M.-H., Shih, Y., 2020. Aerobic degradation and the effect of hexabromocyclododecane by soil microbial communities in Taiwan. Environment International 145, 106128. https://doi.org/10.1016/j.envint.2020.106128

13. Liu, Y., Zhao, C., Lin, D., Lin, H., Lin, Z., 2015. Effect of water extract from spent mushroom substrate after Ganoderma balabacense cultivation by using JUNCAO technique on production performance and hematology parameters of dairy cows: Effect of Hot Water Extract on Dairy Cows. Animal Science Journal n/a-n/a. https://doi.org/10.1111/asj.12371

14. Luan, Z., Sun, G., Huang, Y., Yang, Y., Yang, R., Li, C., Wang, T., Tan, D., Qi, S., Jun, C., Wang, C., Wang, S., Zhao, Y., Jing, Y., 2020. Metagenomics Study Reveals Changes in Gut Microbiota in Centenarians: A Cohort Study of Hainan Centenarians. Front. Microbiol. $11,1474$.

Page $13 / 18$ 
https://doi.org/10.3389/fmicb.2020.01474

15. Marć, M., Bystrzanowska, M., Tobiszewski, M., 2020. Exploratory analysis and ranking of analytical procedures for short-chain chlorinated paraffins determination in environmental solid samples. Science of The Total Environment 711, 134665.

https://doi.org/10.1016/j.scitotenv.2019.134665

16. Moyes, R.B., Reynolds, J., Breakwell, D.P., 2009. Differential Staining of Bacteria: Gram Stain. Current Protocols in Microbiology 15. https://doi.org/10.1002/9780471729259.mca03cs15

17. Pesce, C., Kleiner, V.A., Tisa, L.S., 2019. Simple colony PCR procedure for the filamentous actinobacteria Frankia. Antonie van Leeuwenhoek 112, 109-114. https://doi.org/10.1007/s10482-018-1155-0

18. Reuter, J.A., Spacek, D.V., Snyder, M.P., 2015. High-Throughput Sequencing Technologies. Molecular Cell 58, $586-597$. https://doi.org/10.1016/j.molcel.2015.05.004

19. Rojas-Ruiz, N.E., Sansinenea-Royano, E., Cedillo-Ramirez, M.L., Marsch-Moreno, R., Sanchez-Alonso, P., Vazquez-Cruz, C., 2015. Analysis of Bacillus thuringiensis Population Dynamics and Its Interaction With Pseudomonas fluorescens in Soil. Jundishapur J Microbiol 8. https://doi.org/10.5812/jjm.27953

20. Shahid, I., Han, J., Hardie, D., Baig, D.N., Malik, K.A., Borchers, C.H., Mehnaz, S., 2021. Profiling of antimicrobial metabolites of plant growth promoting Pseudomonas spp. isolated from different plant hosts. 3 Biotech 11, 48. https://doi.org/10.1007/s13205-020-02585-8

21. Vezzulli, L., Stagnaro, L., Grande, C., Tassistro, G., Canesi, L., Pruzzo, C., 2018. Comparative 16SrDNA Gene-Based Microbiota Profiles of the Pacific Oyster (Crassostrea gigas) and the Mediterranean Mussel (Mytilus galloprovincialis) from a Shellfish Farm (Ligurian Sea, Italy). Microb Ecol 75, 495-504. https://doi.org/10.1007/s00248-017-1051-6

22. Woodman, M.E., Savage, C.R., Arnold, W.K., Stevenson, B., 2016. Direct PCR of Intact Bacteria (Colony PCR). Current Protocols in Microbiology 42. https://doi.org/10.1002/cpmc.14

23. Young, B., Delatolla, R., Abujamel, T., Kennedy, K., Laflamme, E., Stintzi, A., 2017. Rapid start-up of nitrifying MBBRs at low temperatures: nitrification, biofilm response and microbiome analysis. Bioprocess Biosyst Eng 40, 731-739. https://doi.org/10.1007/s00449-017-1739-5

24. Zeng, Y., Youssef, M., Wang, L., Alkhars, N., Thomas, M., Cacciato, R., Qing, S., Ly-Mapes, O., Xiao, J., 2020. Identification of Non-Streptococcus mutans Bacteria from Predente Infant Saliva Grown on Mitis-Salivarius-Bacitracin Agar. Journal of Clinical Pediatric Dentistry 44, $28-34$. https://doi.org/10.17796/1053-4625-44.1.5

25. Zhou, J., Chen, S., Shi, W., David-Schwartz, R., Li, S., Yang, F., Lin, Z., 2021. Transcriptome profiling reveals the effects of drought tolerance in Giant Juncao. BMC Plant Biol 21, 2. https://doi.org/10.1186/s12870-020-02785-7

\section{Figures}

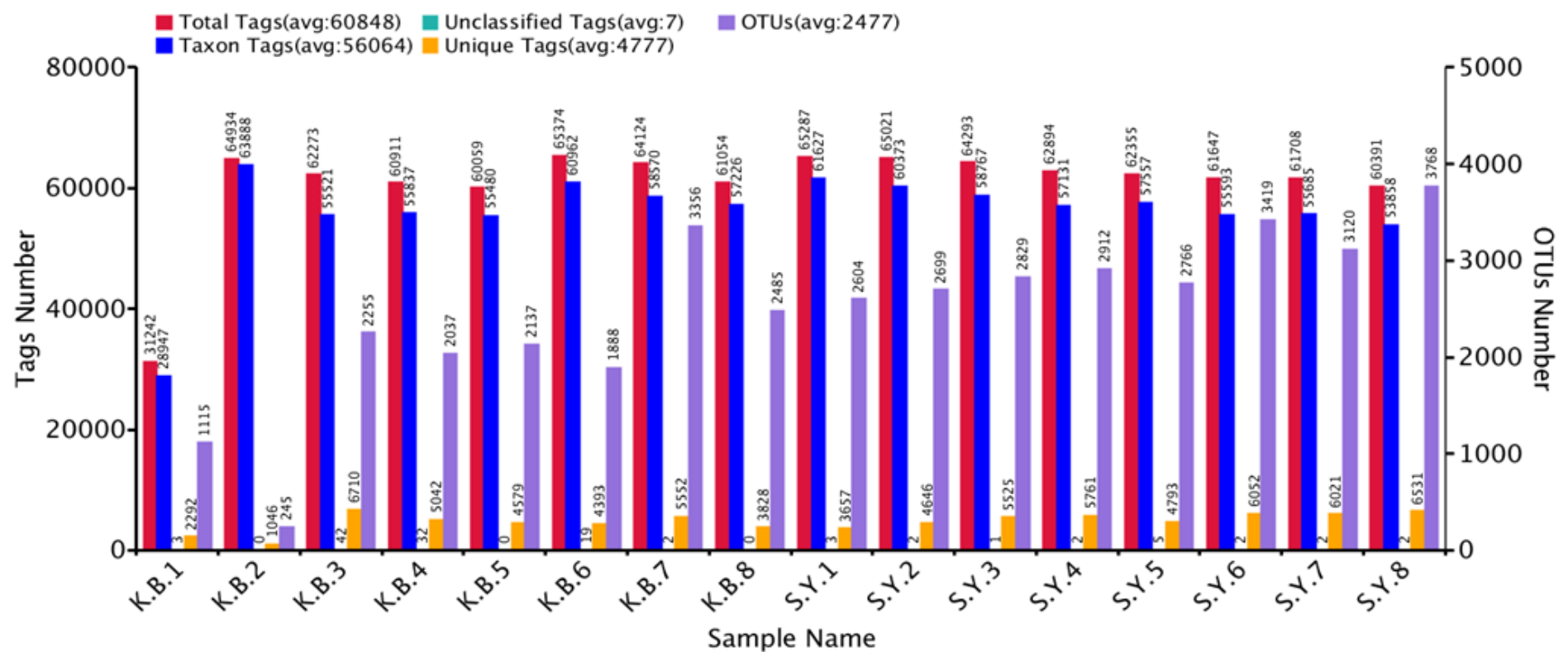

\section{Figure 1}

OTUs cluster quantity diagram of soil microbial population in Blank group and Experimental group 
Figure 2

OTUs clustering numbers of microbial populations at different classification levels in blank group and experimental group soil samples

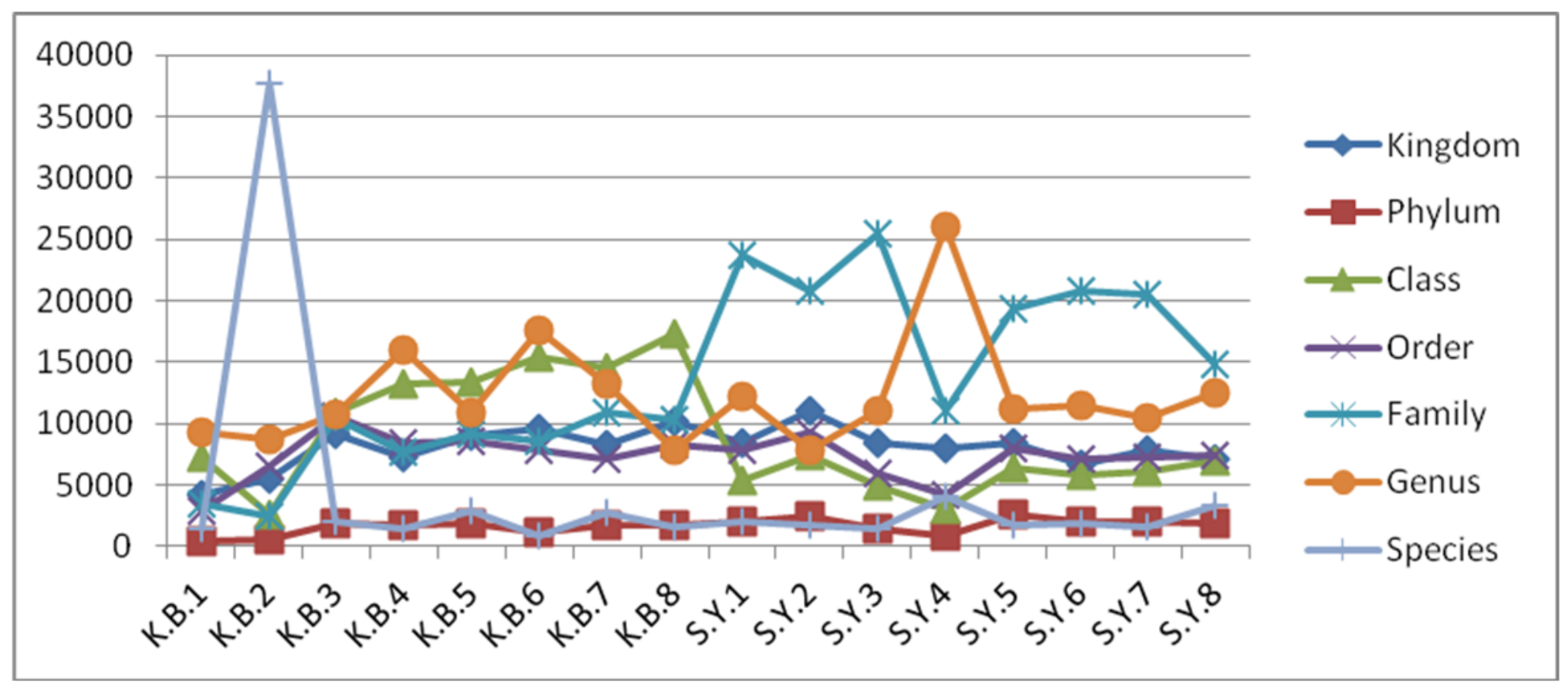

Figure 3

OTUs cluster number curve diagram of microbial populations at different classification levels in blank group and experimental group soil samples

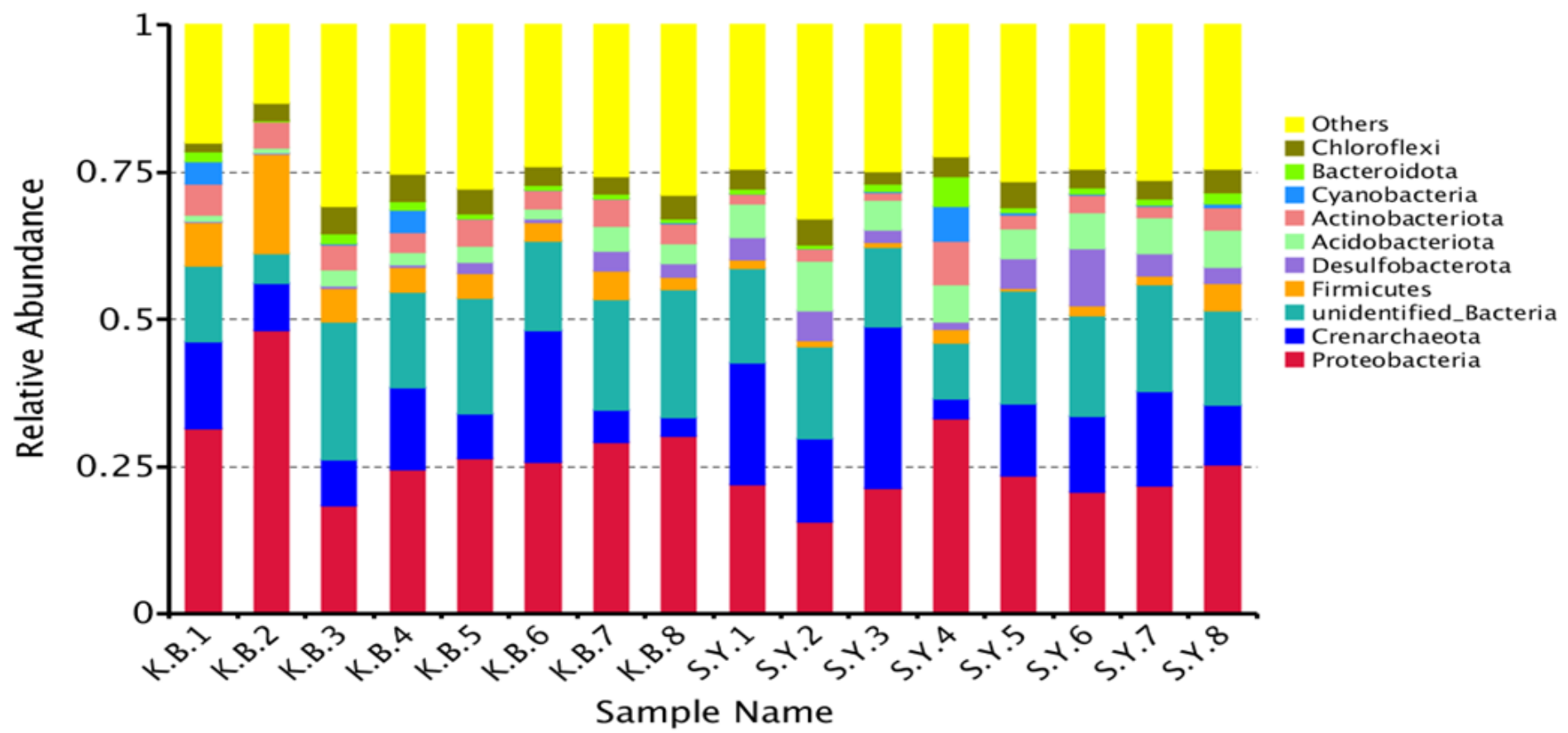

Figure 4

Bar chart of species relative abundance at the level of each Phylum 


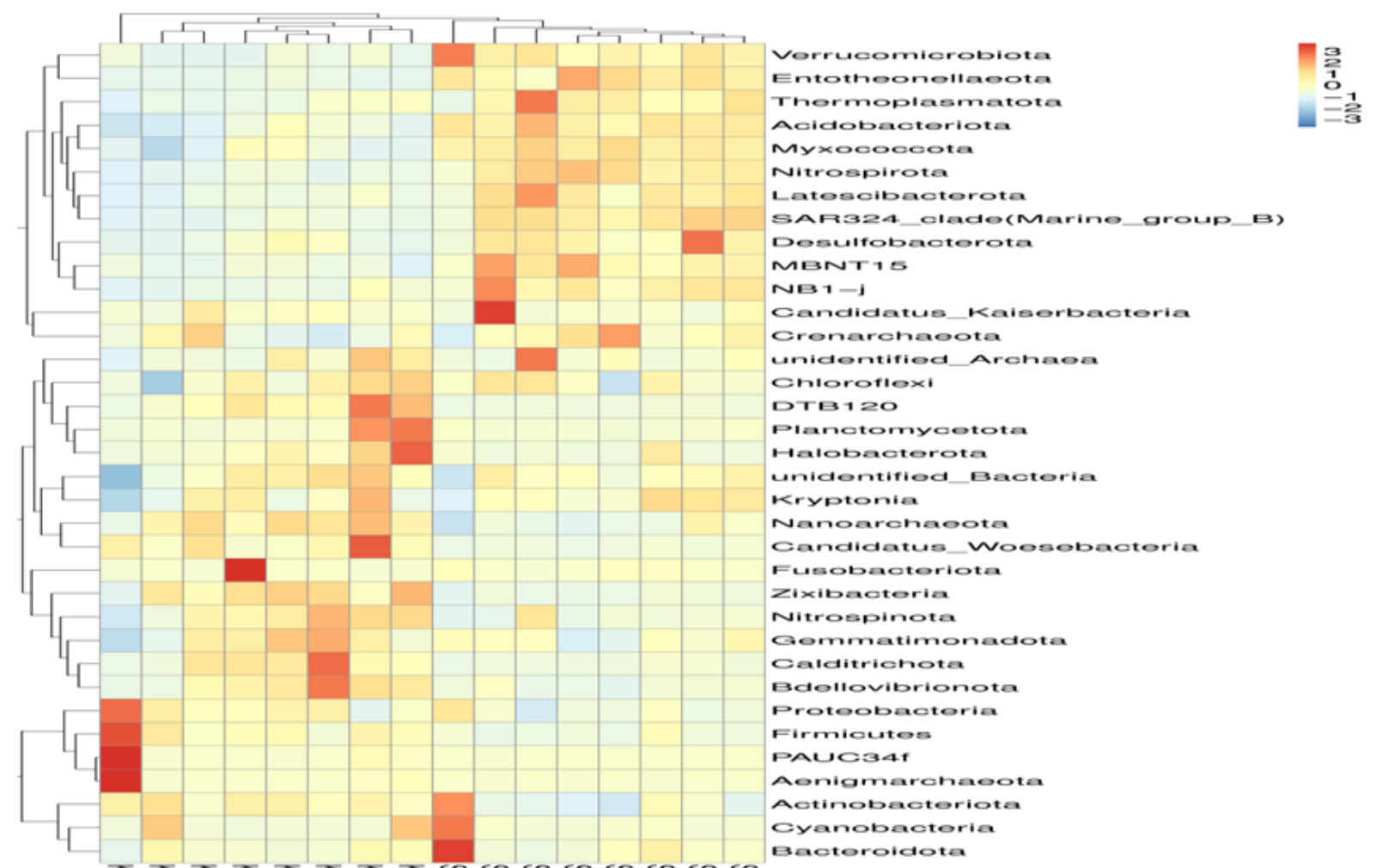

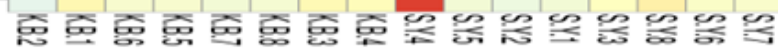

Figure 5

Cluster thermograms of species at the level of each Phylum

\section{Figure 6}

Bar chart of species relative abundance at level of each Order

\section{Figure 7}

Cluster thermograms of species at the level of each Order

\section{Figure 8}

Bar chart of species relative abundance at level of each Family

\section{Figure 9}

Cluster thermograms of species at the level of each Family 
Figure 10

Bar chart of species relative abundance at level of each Genus

\section{Figure 11}

Cluster thermograms of species at the level of each Genus

\section{Figure 12}

Ternaryplot analysis at the classification level of each sample Phylum

\section{Figure 13}

Ternaryplot analysis at the classification level of each sample Order

\section{Figure 14}

Ternaryplot analysis at the classification level of each sample Genus

\section{Figure 15}

Gram staining results of Pseudomonas strain

\section{Figure 16}

Fluorescence production characteristics of Pseudomonas strains

\section{Figure 17}

Gram staining results of Bacillus strain

\section{Figure 18}

Figure of Colony PCR amplification of Pseudomonas fluorescens and Bacillus thuringiensis 
Note: M is the DNA Marker indicator band of DL2000, 1-5 were pseudomonas fluorescens PCR results, and 7-11 were Bacillus thuringiensis PCR results.

Page 18/18 